\title{
Results from the First All-Sky Search for Continuous Gravitational Waves from Small-Ellipticity Sources
}

\author{
Vladimir Dergachev $\odot^{1,2, *}$ and Maria Alessandra Papa $\circledast^{1,2,3, \dagger}$ \\ ${ }^{1}$ Max Planck Institute for Gravitational Physics (Albert Einstein Institute), Callinstrasse 38, 30167 Hannover, Germany \\ ${ }^{2}$ Leibniz. Universität Hannover, D-30167 Hannover, Germany \\ ${ }^{3}$ University of Wisconsin Milwaukee, 3135 North Maryland Avenue, Milwaukee, Wisconsin 53211, USA
}

(Received 17 April 2020; revised 7 July 2020; accepted 17 September 2020; published 22 October 2020)

\begin{abstract}
We present the results of an all-sky search for continuous gravitational-wave signals with frequencies in the 500-1700 Hz range targeting neutron stars with ellipticity of $10^{-8}$. The search is done on LIGO O2 data using the Falcon analysis pipeline. The results presented here double the sensitivity over any other result on the same data [B. P. Abbott et al. (LIGO Scientific Collaboration and Virgo Collaboration), Phys. Rev. D 100, 024004 (2019), C. Palomba et al., Phys. Rev. Lett. 123, 171101 (2019)]. The search is capable of detecting low-ellipticity sources up to $170 \mathrm{pc}$. We establish strict upper limits which hold for worst-case signal parameters. We list outliers uncovered by the search, including several which we cannot associate with any known instrumental cause.
\end{abstract}

DOI: $10.1103 /$ PhysRevLett.125.171101

Introduction.-Continuous gravitational waves are expected over a broad range of frequencies from rapidly rotating compact stars such as neutron stars due to a variety of mechanisms [1] as well as from more exotic scenarios [2-7].

A spinning neutron star that presents a deviation from axisymmetry emits continuous gravitational waves at twice its rotation frequency. Its gravitational-wave brightness is determined by its equatorial ellipticity, which describes how deformed the star is in the plane perpendicular to the rotation axis. Because neutron stars have solar-sized mass packed within a tight radius of $10-15 \mathrm{~km}$, the gravity at their surface is extremely high, making it difficult to support large deformations.

Simulations show that ellipticities as high as $10^{-6}$ can be supported by the crust [8,9], but at present no mechanism is known that mandates the existence of such deformations. Searches so far have failed to find signals from highellipticity sources [10-14].

On the other hand, properties of observed pulsars suggest that a population might exist whose spin evolution is governed by gravitational-wave emission, with typical ellipticities in the range of $\approx 10^{-9}$ to $10^{-8}$ [15]. This is the range targeted by this search (Fig. 1).

We find several outliers and investigate them. We use longer coherence analyses and out-of-sample data. One

Published by the American Physical Society under the terms of the Creative Commons Attribution 4.0 International license. Further distribution of this work must maintain attribution to the author(s) and the published article's title, journal citation, and DOI. Open access publication funded by the Max Planck Society. outlier increases in SNR with an increase in coherence length; however, this increase is not as large as one would expect for an ideal signal on average.

The basic signal assumed at the gravitational-wave detector is a nearly monochromatic signal from an isolated source (i.e., not in a binary), with Doppler modulations due to the relative motion between the source and the detector and amplitude modulations due to the relative orientation of the detector to the source. Optimal sensitivity is typically achieved with a large degree of phase coherence in the signal and for signals matching the assumed waveform model.

No past search has revealed any such signal, over a broad range of possible signal parameters. One reason could be that past searches were not sensitive enough and have not yet probed low enough values of the ellipticity. Another reason (or perhaps an additional reason) might be that the signals deviate enough from the assumed model that the search methods cannot identify them with sufficient confidence.

Deviations from the naive signal model could come about in a number of ways, for example, due to the motion of the source being affected by another body or the signal itself not being perfectly stable. While standard searches might retain very good sensitivity to many of these signals [16], upon closer inspection a signal candidate might be disregarded because it does not pass all the tests that the naive signal would pass.

As more and more sensitive searches yield null results, it seems wise to entertain the possibility that the signals are not quite as we assume. Here we do this by highlighting not only the upper limit results for the standard signals, but also the top outliers that are marginal or inconclusive 


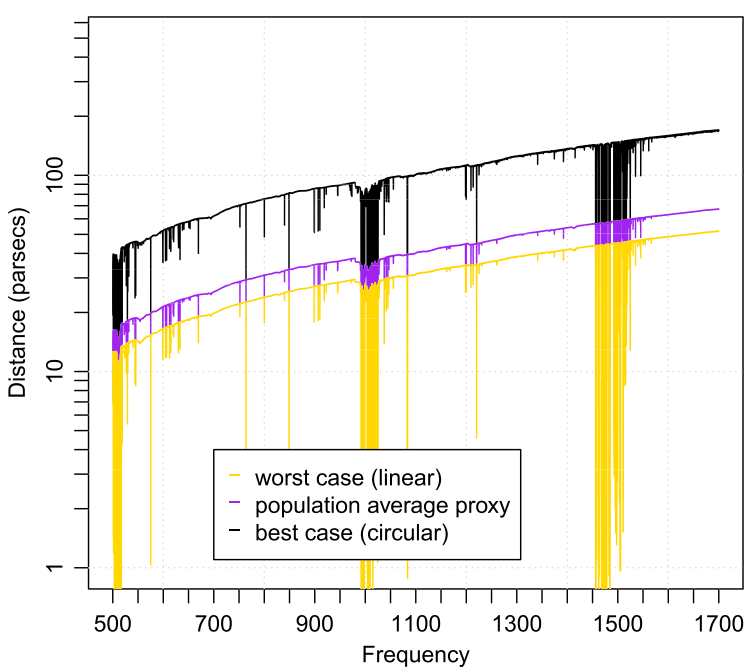

FIG. 1. Range of the search for stars with ellipticity of $10^{-8}$. The $X$ axis shows gravitational-wave frequency, which is twice the pulsar rotation frequency for emission due to an equatorial ellipticity. $R$ modes and other emission mechanisms give rise to emission at different frequencies.

candidates. This will enable cross-checks with data that we have no access to and may lead to the identification of an instrumental or environmental artifact or a signal.

The method.-Broadband all-sky searches for continuous gravitational waves are computationally challenging. The most sensitive surveys employ multistage searches that rely on clever search methods, computationally efficient algorithms, and much computing power.

A key characteristic of long-duration searches is the employed coherence length. Increasing the coherence length increases the search sensitivity and makes the search more selective, being able to distinguish more finely different signal parameters like sky location and frequency. The number of separate points of parameter space that need to be explored grows with the increasing coherence length, and this generates greater computational demands. The computational cost can scale as the fourth power of the coherence length for even simple searches, and it grows very quickly for searches taking into account more complex signals, with higher-order frequency derivatives, or in the presence of a companion object in a binary system.

A common way to construct a search with a given coherence length is to partition the data into segments spanning that coherence length, perform a matched filter analysis on each piece, and then combine the results. This is known as a "semicoherent" search, because the results of the coherent matched filter analysis are combined with no phase information. See [13,14,17-19], and references therein for an overview and comparison of the different families of semicoherent search methods.

Loosely coherent methods [20-22] are constructed by partitioning the parameter space of the searched signals into families of closely related signals that can be considered to be perturbations of each other. The search is then designed to work on each family separately, and, because many signals are searched for "at the same time," it leverages significant economy of scale.

The newest loosely coherent implementation targeting longer coherent timescales, the Falcon search, represents a breakthrough in performance and sensitivity [13]. We have explored the LIGO O1-run data employing the Falcon search with a first-stage coherence length of $4 \mathrm{~h}$ and demonstrated its performance and computational efficiency $[10,13]$. In this paper, we push the envelope further, doubling the size of the input data, tripling the coherence length of the first stage, extending the frequency range by a factor of nearly 3 , and searching high frequencies. (The necessary computing power scales as the cube of highest frequency searched.) We attain more than a twofold increase in strain sensitivity with respect to the best LIGO all-sky search on the same data (Fig. 2).

The search.-The computational load of an all-sky search per-hertz-searched is massively different in the kilohertz region and in the $O(100) \mathrm{Hz}$ region. This means that, in order to achieve optimal sensitivity, different search setups should be used in different frequency ranges (this argument is clearly illustrated in Refs. [23,24]). We choose here to tackle the challenging high-frequency region.

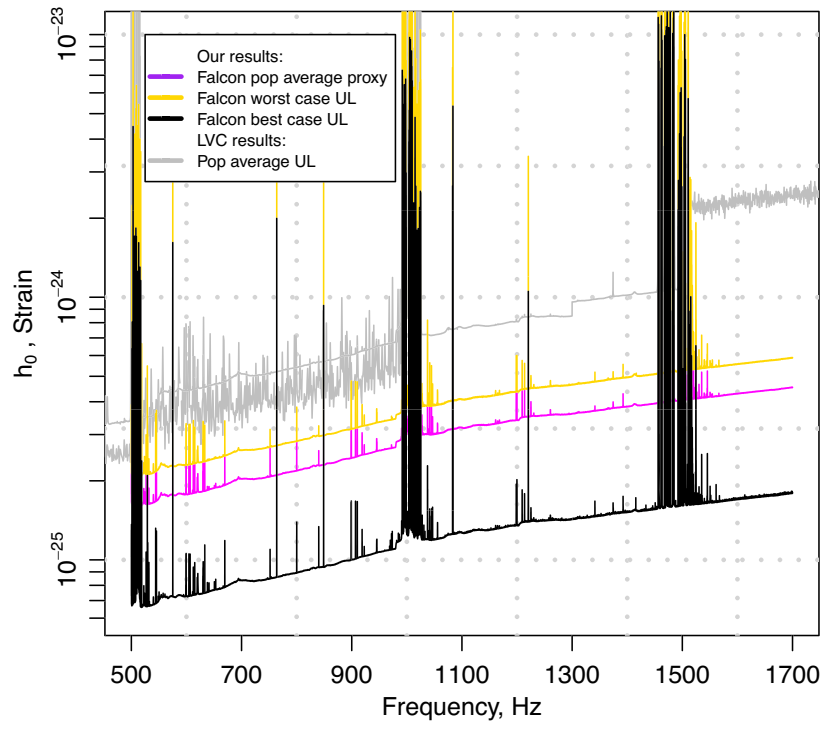

FIG. 2. The intrinsic gravitational-wave strain-amplitude upper limit (vertical axis) is plotted against the signal frequency. The lower curve (black) shows the best-case upper limits (circularly polarized signals), the next curve (purple) shows the populationaverage proxy, and then (in yellow) we have the worst-case upper limits. The fainter lines at the top are the upper limits from the latest LIGO-Virgo Collaboration (LVC) all-sky survey [12], and they can be directly compared to our population-average ones (middle purple curve). The LVC results are produced independently by three different pipelines which cover overlapping frequency ranges. 
We aim at detecting continuous gravitational-wave signals with a frequency between 500 and $1700 \mathrm{~Hz}$ and first-order frequency derivative $\left|f_{1}\right| \leq 3 \times 10^{-12} \mathrm{~Hz} / \mathrm{s}$ for frequencies below $1000 \mathrm{~Hz}$ and $\leq 2.5 \times 10^{-12} \mathrm{~Hz} / \mathrm{s}$ above $1000 \mathrm{~Hz}$. The spindown range is consistent with our ellipticity target at a level $<10^{-8}$. It is also generous: The largest known frequency drift among pulsars spinning faster than $250 \mathrm{~Hz}$ is $-1.73 \times 10^{-13} \mathrm{~Hz} / \mathrm{s}$, for $\mathrm{J} 1824-2452 \mathrm{~A}$ spinning at $327 \mathrm{~Hz}$ [25]. The full search parameter range is given in Table II.

We use the public LIGO [26] O2 data [27,28]. Below $1000 \mathrm{~Hz}$, these data were subject to a cleaning procedure that removed a substantial amount of spurious instrumental noise [29]. In general, this procedure will contribute an additional systematic uncertainty to the calibration, which is, however, not stated. We use the available $\mathrm{O} 2$ data as is; thus, our results below $1000 \mathrm{~Hz}$ have a potential systematic uncertainty that overall should match the uncertainty (5\% and $10 \%$ for $\mathrm{H} 1$ and $\mathrm{L} 1$, respectively) given in LIGO papers such as Ref. [12].

The search begins with a coherence length of $12 \mathrm{~h}$ that is extended to 6 days through three follow-up stages (Table I). The coherence length increases from stage to stage, and with it the search sensitivity also increases. Candidates pass to the next stage only if their detection statistic value exceeds a predetermined threshold value, which increases from one stage to the next. The last stage also includes consistency checks between the single-instrument results. Candidates that survive this entire sequence of tests represent one of the results of the search. We refer to these as the outliers from the search. Every outlier is defined by a set of signal parameters (frequency, sky position, and frequency derivative) and signal to noise ratio value (SNR).

Upper limit results. - The other results of this paper are the $95 \%$ confidence-level continuous gravitational-wave amplitude upper limits $h_{0}$. These represent the smallest amplitude of a continuous signal with a given frequency, coming from an arbitrary direction in the sky and with frequency drift in our search range, that we can exclude from impinging on the detectors. We have assumed a quasimonochromatic signal with slow evolution in frequency that can be approximated by a quadratic model:

$$
f(t)=f_{0}+\left(t-t_{0}\right) f_{1}+\left(t-t_{0}\right)^{2} f_{2} / 2,
$$

TABLE I. Parameters for each stage of the search. The stage-3 outliers are subject to an additional consistency check.

\begin{tabular}{lcc}
\hline \hline Stage & Coherence length (hours) & Minimum SNR \\
\hline 0 & 12 & 6 \\
1 & 24 & 7 \\
2 & 48 & 8 \\
3 & 144 & 16 \\
\hline \hline
\end{tabular}

where $f_{0}$ is the signal frequency at GPS epoch $t_{0}=$ 1183375935 and $f_{1}$ and $f_{2}$ are the linear and quadratic frequency drifts, respectively.

Our upper limits are established based on the observed power estimates and are valid even in the most heavily contaminated spectral regions. We use a procedure based on the so-called "universal statistics" [30] which determines the smallest gravitational-wave intrinsic signal amplitude $h_{0}$ consistent with the observed power. This produces valid upper limits without assumptions on the shape of the noise distribution. By construction, the upper limits also hold in the presence of a signal.

We compute three upper limits: the so-called worst-case upper limit, which is the largest upper limit over those established for individual sky locations, polarization (maximum over polarization is usually reached for linear polarizations), spindown, and frequency; the circularpolarization upper limit, which is the largest upper limit over those established for individual sky locations, spindown, and frequency, having assumed circular polarization of the signal; and, finally, a proxy for the populationaverage upper limit which is determined over a population of sources uniformly distributed on the sky and with all possible polarizations [uniform in the cosine of the inclination angle $(\cos \imath)$ and polarization angle $\psi]$. This last upper limit is provided for ease of comparison with other search results [11,14,31,32], and it is estimated (hence, it is a proxy) as a weighted average of the upper limits from the individual polarizations.

The population-average proxy is verified by directly measuring the detection efficiency of fake signals added to the real data with frequencies in the sample bands 500-600 and 1075.40-1100 Hz. Each fake signal has an intrinsic strain amplitude $h_{0}$ equal to the population-average upperlimit proxy value from its frequency band; other parameters are uniformly distributed in the searched parameter space, apart for the spindown values that are log-uniformly distributed in the search range. The full analysis is performed for each fake signal, covering the entire sky and including outlier follow-up. A fake signal is considered detected when an outlier is found within $5 \times 10^{-5} \mathrm{~Hz}$ of the signal frequency $f$, within $10^{-12} \mathrm{~Hz} / \mathrm{s}$ of its spindown, and within $0.06 \mathrm{~Hz} / f$ radians of its sky location, the latter calculated after projecting on the ecliptic plane ("ecliptic distance"). We obtain 95\% recovery of injections everywhere except the heavily contaminated violin mode region, and even in those bands the detection efficiency does not drop lower than $90 \%$.

The upper limits are plotted in Fig. 2. The upper limit data are available in computer-readable format in Ref. [33].

Assuming an optimally oriented source of ellipticity $10^{-8}$, we are sensitive as far as $170 \mathrm{pc}$ at $1700 \mathrm{~Hz}$. Figure 1 shows the reach of the search to such sources. Above $700 \mathrm{~Hz}$, with the exception of a few contaminated frequency bands, we can exclude the presence of such sources 
TABLE II. Signal-parameter space covered in this search. Both positive and negative values of first- and second-order frequency derivatives $f_{1}$ and $f_{2}$ are explored.

\begin{tabular}{|c|c|c|}
\hline Band $[\mathrm{Hz}]$ & $\left|f_{1}\right|_{\max } 10^{-12}[\mathrm{~Hz} / \mathrm{s}]$ & $\left|f_{2}\right|_{\max } 10^{-20}\left[\mathrm{~Hz} / \mathrm{s}^{2}\right]$ \\
\hline $500-1000$ & 3 & 4 \\
\hline $1000-1700$ & 2.5 & 2 \\
\hline
\end{tabular}

(aside from the outliers listed in Supplemental Material [33]) within a $20 \mathrm{pc}$ distance from Earth.

It is reasonable to expect neutron stars from our target population within this range: Assuming a galactic population of $10^{9}$ neutron stars, if the density in space of neutron stars is the same as that of the known pulsars, then within $20 \mathrm{pc}$ of Earth we expect (this conservative estimate assumes spherical distribution of neutron stars; since $1.36 \mathrm{kpc}$ is large compared to the thickness of the Milky Way disk and size of galactic arms, we expect that the true number is much larger) to have as many neutron stars, and we observe as pulsars in $\sim 1.36 \mathrm{kpc}$. At the time of writing the ATNF catalog [25] shows 56 pulsars rotating above $250 \mathrm{~Hz}$ within $1.36 \mathrm{kpc}$ of Earth, and of these 12 are isolated.

Our upper limits are also relevant for boson condensates around black holes [3,4], which are expected to emit monochromatic continuous wave signals [34]. Indeed, if ultralight bosons exist, they will form clouds around rotating black holes via superradiance instability and through annihilations and level transitions will emit continuous gravitational waves. We leave it to the interested reader to constrain from our upper limits physical quantities of interest, based on the specific model they wish to consider. Assuming the ensemble signal of Ref. [35] from a galactic population of $O\left(10^{8}\right)$ isolated stellar mass black holes with maximum mass $30 M_{\odot}$ and maximum initial spin uniformly distributed in $[0,1]$, our results exclude bosons with masses in the range $\approx[1.0-3.0] \times 10^{-12} \mathrm{eV}$.

Outliers.-The full list of outliers is available in Ref. [33]. Supplemental Material [33] also includes a summary table listing the largest SNR outlier in every

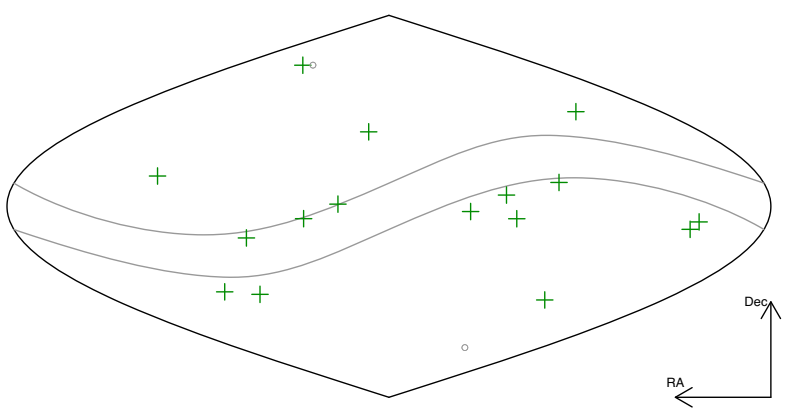

FIG. 3. Location in the sky of the outliers not associated to any instrumental disturbance. The wide band shows the ecliptic plane, and the two small circles are the ecliptic poles.
$0.1 \mathrm{~Hz}$ band. This is especially helpful for the loud fake signals present in the data stream for validation purposes [36], since these produce many outliers. Because these fake signals are produced directly at the LIGO detectors by an appropriate excitation of its mirrors, they are commonly referred to as "hardware injections." For convenience, we give the parameters of these fake signals in Supplemental Material [33].

Our top eight outliers are caused by such hardware injections. The large majority of the remaining outliers are due to large hardware artifacts. There remain outliers whose origin could perhaps be identified based on data from physical and environmental monitoring channels. Since these data are not public, we cannot perform such investigations.

A key question is whether any of the listed outliers are produced by an astrophysical source. The simulations performed to verify the population average proxy assure us that if a signal were present in the data with detectable strength, there would be a corresponding outlier. Clearly, none of the outliers due to hardware injections are astrophysical. A number of outliers are not due to hardware-injected signals but are located in frequency bands with instrumental contamination. As the pipeline is perfectly able to detect signals in most heavily contaminated bands, this does not rule out these outliers. However, it does cast doubt on their significance, as contamination can artificially increase the SNR in a single or both instruments.

The 16 outliers not associated with any known instrumental disturbance are the most interesting. Their sky locations are shown in Fig. 3. They do not appear to

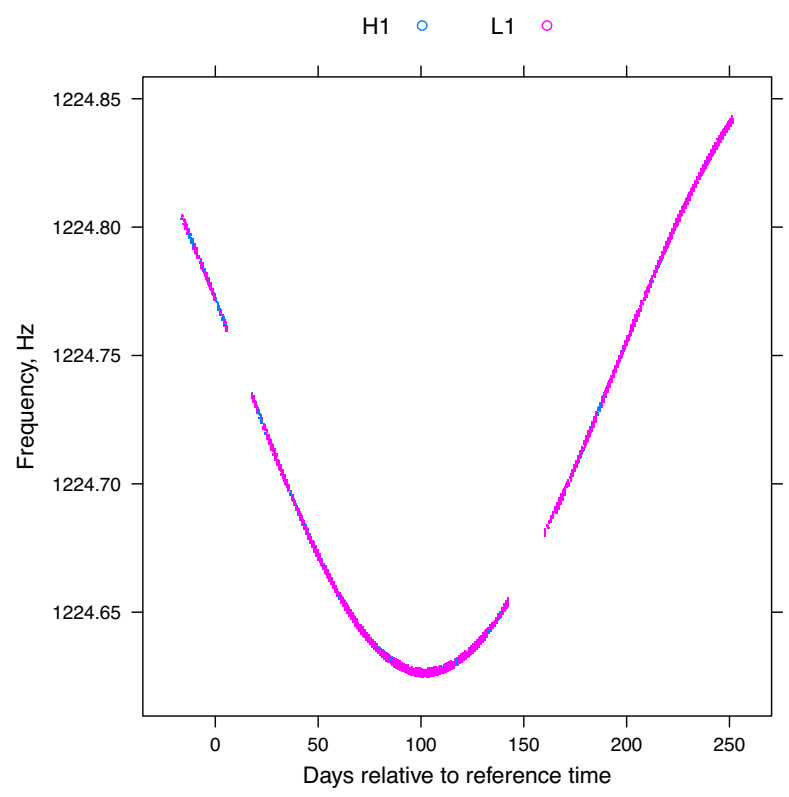

FIG. 4. Apparent frequency of a signal with parameters equal to those of outlier 43 at $1224.74 \mathrm{~Hz}$, at the detectors. The difference in Doppler shifts between interferometers is small compared to the Doppler shifts from Earth's orbital motion. 


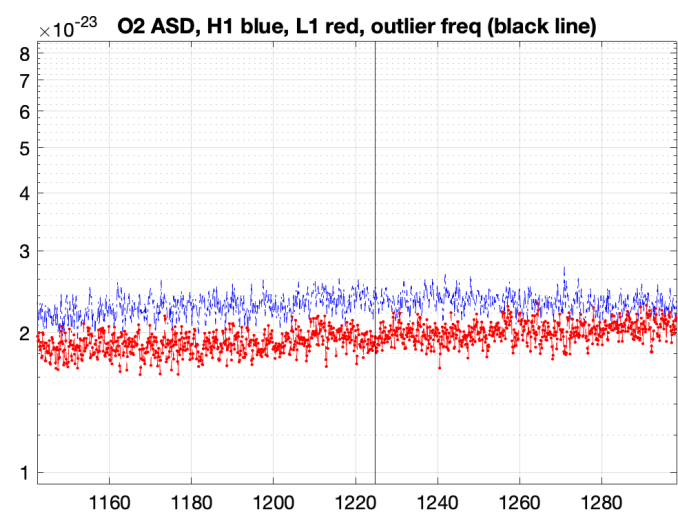

FIG. 5. Average amplitude spectral density around the frequency of outlier 43 .

cluster in any location tied to instrumental artifacts, such as the ecliptic pole. If the outliers were due to astrophysical signals that follow the frequency model of Eq. (1), then the uncertainty on their true location would be within the limits established by the Monte Carlo simulations discussed above, i.e., $0.06 \mathrm{~Hz} / f \mathrm{rad}$, projected on the ecliptic plane.

The frequency spectrum of the data around these outliers is manually examined, and none reveals any obvious contamination. As an example we show plots (Figs. 4 and 5) corresponding to the highest-frequency outlier 43 at $1224.75 \mathrm{~Hz}$. The apparent frequency of a signal with the parameters of outlier 43 spans almost $0.25 \mathrm{~Hz}$ during the $\mathrm{O} 2$ observing time, and its evolution is shown in Fig. 4.

The other 15 outliers have similar characteristics. The frequency evolution curves change depending on the outlier parameters, but they all exhibit an appreciable modulation.

Ad hoc investigations are focused on outliers 25 and 43, which are consistently found with both the Falcon and semicoherent $F$-statistic analyses. Follow-ups over longer coherence lengths (up to $500 \mathrm{~h}$ ) suggest that the most promising outlier is outlier 25 . We note that the $f_{1}$ value of outlier 25 is positive, which would mean that, if the outlier were associated to an astrophysical continuous signal, what is observed is not directly related to the spin frequency of the star.

The SNR buildup for outlier 25 over searches with an increasingly long coherent time baseline is not inconsistent with what is expected from a signal. This holds true also for the fully coherent search over the $\mathrm{O} 2$ data, albeit the recovered SNR is lower than the expectation value based on the previous stages and with indications that the coherence might not be completely stable [the most significant measured $2 \mathcal{F}$ value is $\approx 47 ; 2 \mathcal{F}$ is the optimal multidetector fully coherent detection statistic [38] for signals of the type of Eq. (1)]. A follow-up on $\mathrm{O} 1$ data is inconclusive: The detection statistic value is not inconsistent with the expectations under the signal hypothesis, and the Gaussian-noise $p$ value is $\lesssim 1 \%$.
As already pointed out, we are unable to investigate the $\mathrm{O} 1$ and $\mathrm{O} 2$ instrumental data channels, as these data are not part of either the $\mathrm{O} 1$ or $\mathrm{O} 2$ public data releases.

A first detection can be á la GW150914 (the first gravitational-wave signal [37]) with a single loud event that spectacularly meets all the predictions; a less glamorous scenario is that evidence builds up over time with outliers first identified in a broad survey like this and later consistently recovered on new data or through the identification of an electromagnetic counterpart. In this spirit, we report and discuss the outliers that we find.

Our outliers are not present in the outlier or subthreshold candidate lists from earlier papers. This is not surprising, because previous searches on LIGO $\mathrm{O} 2$ data that cover the parameter space of this search $[12,31]$ are significantly less sensitive.

The $\mathrm{O} 3$ data are roughly a factor of 2 more sensitive than the $\mathrm{O} 2$ data employed in this search, so the LVC broadband searches should be able to probe continuous gravitationalwave amplitudes comparable to these. Also, targeted searches based on the information presented here, which are comparatively less complex than these broad surveys, should be straightforward. We look forward to comparing our results on $\mathrm{O} 3$ data with those from the LVC, and we are ready to perform fast-turnaround investigations on new data.

The search was performed on the ATLAS cluster at AEI Hannover. We thank Bruce Allen, Carsten Aulbert, and Henning Fehrmann for their support. We also acknowledge useful discussion with Bruce Allen on the distribution of nearby neutron stars. This research has made use of data, software, and/or web tools obtained from the LIGO Open Science Center, a service of LIGO Laboratory, the LIGO Scientific Collaboration and the Virgo Collaboration. LIGO is funded by the U.S. National Science Foundation. Virgo is funded by the French Centre National de Recherche Scientifique (CNRS), the Italian Istituto Nazionale della Fisica Nucleare (INFN), and the Dutch Nikhef, with contributions by Polish and Hungarian institutes.

*vladimir.dergachev@aei.mpg.de †maria.alessandra.papa@aei.mpg.de

[1] P. D. Lasky, Gravitational waves from neutron stars: A review, Pub. Astron. Soc. Aust. 32, e034 (2015).

[2] R. Brito, S. Ghosh, E. Barausse, E. Berti, V. Cardoso, I. Dvorkin, A. Klein, and P. Pani, Gravitational wave searches for ultralight bosons with LIGO and LISA, Phys. Rev. D 96, 064050 (2017).

[3] M. Baryakhtar, R. Lasenby, and M. Teo, Black hole superradiance signatures of ultralight vectors, Phys. Rev. D 96, 035019 (2017).

[4] A. Arvanitaki, M. Baryakhtar, R. Lasenby, S. Dimopoulos, and S. Dubovsky, Black hole mergers and the QCD axion at advanced LIGO, Phys. Rev. D 95, 043001 (2017). 
[5] A. Arvanitaki, M. Baryakhtar, and X. Huang, Discovering the QCD Axion with black holes and gravitational waves, Phys. Rev. D 91, 084011 (2015).

[6] C. J. Horowitz and S. Reddy, Gravitational Waves from Compact Dark Objects in Neutron Stars, Phys. Rev. Lett. 122, 071102 (2019).

[7] C. J. Horowitz, M. A. Papa, and S. Reddy, Gravitational waves from compact dark matter objects in the solar system, Phys. Lett. B 800, 135072 (2020).

[8] C. J. Horowitz and K. Kadau, Breaking Strain of Neutron Star Crust and Gravitational Waves, Phys. Rev. Lett. 102, 191102 (2009).

[9] N. K. Johnson-McDaniel and B. J. Owen, Maximum elastic deformations of relativistic stars, Phys. Rev. D 88, 044004 (2013).

[10] V. Dergachev and M. A. Papa, Results from an extended Falcon all-sky survey for continuous gravitational waves, Phys. Rev. D 101, 022001 (2020).

[11] B. P. Abbott et al. (LIGO Scientific Collaboration and Virgo Collaboration), Full band all-sky search for periodic gravitational waves in the O1 LIGO data, Phys. Rev. D 97, 102003 (2018).

[12] B. P. Abbott et al. (LIGO Scientific Collaboration and Virgo Collaboration), All-sky search for continuous gravitational waves from isolated neutron stars using advanced LIGO O2 data, Phys. Rev. D 100, 024004 (2019).

[13] V. Dergachev and M. A. Papa, Sensitivity Improvements in the Search for Periodic Gravitational Waves Using O1 LIGO Data, Phys. Rev. Lett. 123, 101101 (2019).

[14] B. P. Abbott et al. (LIGO Scientific and Virgo Collaborations), First low-frequency Einstein@Home all-sky search for continuous gravitational waves in Advanced LIGO data, Phys. Rev. D 96, 122004 (2017).

[15] G. Woan, M. D. Pitkin, B. Haskell, D. I. Jones, and P. D. Lasky, Evidence for a minimum ellipticity in millisecond pulsars, Astrophys. J. Lett. 863, L40 (2018).

[16] A. Singh, M. A. Papa, and V. Dergachev, Characterizing the sensitivity of isolated continuous gravitational wave searches to binary orbits, Phys. Rev. D 100, 024058 (2019).

[17] S. Walsh, K. Wette, M. A. Papa, and R. Prix, Optimizing the choice of analysis method for all-sky searches for continuous gravitational waves with Einstein@ Home, Phys. Rev. D 99, 082004 (2019).

[18] S. Walsh et al., Comparison of methods for the detection of gravitational waves from unknown neutron stars, Phys. Rev. D 94, 124010 (2016).

[19] C. Dreissigacker, R. Prix, and K. Wette, Fast and accurate sensitivity estimation for continuous-gravitational-wave searches, Phys. Rev. D 98, 084058 (2018).

[20] V. Dergachev, On blind searches for noise dominated signals: A loosely coherent approach, Classical Quantum Gravity 27, 205017 (2010).

[21] V. Dergachev, Loosely coherent searches for sets of wellmodeled signals, Phys. Rev. D 85, 062003 (2012).
[22] V. Dergachev, Loosely coherent searches for medium scale coherence lengths, Phys. Rev. D 85, 062003 (2012).

[23] J. Ming, B. Krishnan, M. A. Papa, C. Aulbert, and H. Fehrmann, Optimal directed searches for continuous gravitational waves, Phys. Rev. D 93, 064011 (2016).

[24] J. Ming, M. A. Papa, B. Krishnan, R. Prix, C. Beer, S. J. Zhu, H. B. Eggenstein, O. Bock, and B. Machenschalk, Optimally setting up directed searches for continuous gravitational waves in Advanced LIGO O1 data, Phys. Rev. D 97, 024051 (2018).

[25] R. N. Manchester, G. B. Hobbs, A. Teoh, and M. Hobbs, The Australia telescope national facility pulsar catalogue, Astron. J. 129, 1993 (2005).

[26] J. Aasi et al. (LIGO Scientific Collaboration), Advanced LIGO, Classical Quantum Gravity 32, 074001 (2015).

[27] M. Vallisneri, J. Kanner, R. Williams, A. Weinstein, and B. Stephens The LIGO Open Science Center, J. Phys. Conf. Ser. 610, 012021 (2015).

[28] LIGO Open Science Center, https://doi.org/10.7935/CA75FM95 (2019)

[29] J. C. Driggers et al. (LIGO Scientific Collaboration Instrument Science Authors), Improving astrophysical parameter estimation via offline noise substraction for advanced LIGO, Phys. Rev. D 99, 042001 (2019).

[30] V. Dergachev, A novel universal statistic for computing upper limits in ill-behaved background, Phys. Rev. D 87, 062001 (2013).

[31] C. Palomba et al., Direct Constraints on Ultra-Light Boson Mass from Searches for Continuous Gravitational Waves, Phys. Rev. Lett. 123, 171101 (2019).

[32] B. P. Abbott et al. (LIGO Scientific Collaboration and Virgo Collaboration), All-sky search for periodic gravitational waves in the O1 LIGO data, Phys. Rev. D 96, 062002 (2017).

[33] See Supplemental Material at http://link.aps.org/ supplemental/10.1103/PhysRevLett.125.171101 for numerical values of upper limits, outlier tables, and hardware-injection parameters. Also at https://www.aei.mpg.de/ continuouswaves/O2Falcon500-1700.

[34] A. Arvanitaki, M. Baryakhtar, and X. Huang, Discovering the QCD axion with black holes and gravitational waves, Phys. Rev. D 91, 084011 (2015).

[35] S. J. Zhu, M. Baryakhtar, M. A. Papa, D. Tsuna, N. Kawanaka, and H. Eggenstein, Characterizing the continuous gravitational-wave signal from boson clouds around Galactic isolated black holes, Phys. Rev. D 102, 063020 (2020).

[36] $\mathrm{O} 2$ continuous hardware injection signal parameters, https:// www.gw-openscience.org/O2_injection_params.

[37] B. P. Abbott et al. (LIGO Scientific and Virgo Collaborations), Observation of Gravitational Waves from a Binary Black Hole Merger, Phys. Rev. Lett. 116, 061102 (2016).

[38] C. Cutler and B.F. Schutz, The generalized F-statistic: Multiple detectors and multiple GW pulsars, Phys. Rev. D 72, 063006 (2005). 